\title{
Patient-controlled TENS reduces the discomfort of ID nerve block.
}

\section{Meechan J G, Gowans A J, Welbury R R. The use of patient-controlled TEN S to decrease the discomfort of regional anaesthesia in dentistry: a randomised controlled clinical trial. J of Dent. 1998; 26: 417-420}

Objective To compare the use of topical anaesthesia and transcutaneous electronic nerve stimulation (TENS) as a means of reducing the discomfort of inferior dental nerve block injection.

Design A randomised controlled trial in a UK dental school.

Intervention 100 adult patients requiring lower tooth extraction that necessitated long buccal and inferior alveolar block anaesthesia were randomly assigned to 3 groups. The same operator using the same type of anaesthetic for each case administered all injections. Each patient received a long buccal (LB) injection. They then received an inferior alveolar injection (IDB) and lingual block after either - no mucosal preparation, $20 \%$ topical benzocaine or TENS treatment.

Outcome measures Patients and scored their discomfort on a visual analogue scale (VAS) following the administration of both the LB and IDB injections. The statements 'no pain' and 'unbearable pain' anchored the VAS.

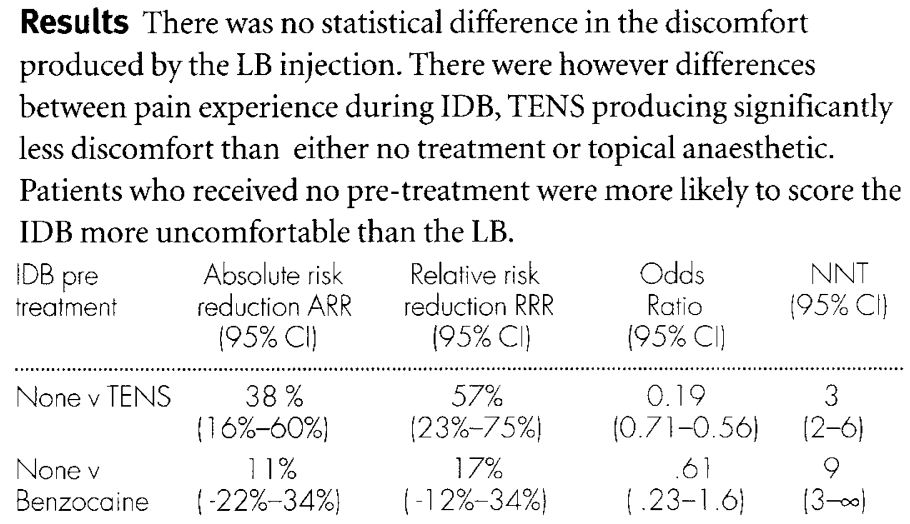

Conclusion Patient-controlled TENS reduced but did not eliminate discomfort experienced during inferior dental block and lingual block compared with $20 \%$ benzocaine topical anaesthetic.

Address for reprints: Department of Oral and Maxillofacial Surgery, The Dental Hospital, Framlington Place, Newcastle upon Tyne, NE2 4BW, UK.

\section{Commentary}

The authors carried out a study on the effects of different pre-treatments on the discomfort of intra-oral regional block anaesthesia which is an extremely common cause of anxiety in the dental office. This is a complex area of study and a number of methodological issues arose. 100 adult patients were studied but no indication is given that these patients were randomly or consecutively identified nor that they were a homogenous group in terms of their need for extraction of a lower tooth, i.e. acute or elective extractions, simple or surgical extractions. These variables could be relevant as the experience of pain has an important emotional content. The patients were randomly assigned to three groups - no pre-treatment, application of topical anaesthetic and application of TENS for the inferior dental block (IDB) injection.

The study injection was preceded by a long buccal (LB) injection. It could be helpful to know if bone was encountered during the LB injection and whether a new needle was used for the study injection. A two minute delay was incorporated into the no pre-treatment group to account for two minutes of topical anaesthetic application. No note is made of the time required to set up the TENS machine and whether this delay and the activity of preparation had any effect on the reporting of pain. Does the IDB technique allow for a superficial injection of local anaesthesia before the needle is advanced into deeper tissues? The sole use of visual analogue scale to assess the multidimensional nature of pain is problematic and may not accurately reflect the complexity of the pain experience. ${ }^{1}$ An adjectival scale derived from the McGill Pain Questionnaire and validated by cross modality matching, which reflects sensory, affective and reactive components, could overcome the problem. ${ }^{2}$

Despite these limitations, there was no statistical difference in the discomfort produced by $\mathrm{LB}$ injection suggesting that this was a suitable baseline measurement. Topical anaesthesia did not affect the pain experience for the IDB injection compared to no pre-treatment. TENS reduced but did not eliminate the pain.

From a statistical point of view, the absolute risk reduction (ARR) is 38\% for TENS and $11 \%$ for topical anaesthesia. The number needed to treat (NNT) to see the benefit of TENS is three and for topical anaesthesia is nine. From a clinical perspective, it seems unlikely that these results will change practice habits. The momentary discomfort of an injection has to be weighed against the overall benefit produced and the other components of the global dental experience.

1 Reading A E. Testing pain mechanisms in persons in pain. 195-204.In Textbook of Pain (Ed) Wall PD and Melzack R. Ch irchill Livingstone, Edinburgh. 1987.

2 Tursky B. The development of a pain perception profile: a psychophysical approach. 171 In Pain, new perspectives in therapy and research (eds) Weisenberg $M$, Tursky B. Plenum Press, New York 1976.

\section{Dr David Keith}

Department of Oral and Maxillofacial Surgery, Massachusetts General Hospital Boston USA 